\title{
Author Index for Volume 69
}

\begin{tabular}{|c|c|c|c|}
\hline Abdelfattah, MS & 446 & Cai, Q & 7 \\
\hline Adachi, $\mathrm{H}$ & 893 & Caillon, J & 8 \\
\hline Aguilar, J & 353 & Cane, DE & 473,5 \\
\hline Ahn, JS & 886 & Cao, L & \\
\hline Aihara, Y & 299 & Chang, A-Y & \\
\hline Ajito, K & $191,368,428,440$ & Chang, C & \\
\hline Akagawa, KS & 319 & Chang, C-Y & \\
\hline Akamatsu, Y & 893 & Che, Q & \\
\hline Akimoto, M & 187 & Che, Y & \\
\hline Aksoy, SÇ & 51 & Cheah, S-E & \\
\hline Alexander, BK & 567 & Chen, A-1 & \\
\hline Amemiya, M & 176 & Chen, L & \\
\hline An, Z & 141 & Chen, $\mathrm{R}$ & \\
\hline Ando, $\mathrm{O}$ & 747,754 & Chen, X & 773,8 \\
\hline Anzai, Y & 395 & Chen, Y & \\
\hline Arai, S & 340 & Chen, Z & \\
\hline Arimoto, $\mathrm{H}$ & 203 & Chida, N & \\
\hline Asakawa, T & 299 & Chmielewski, M & \\
\hline \multirow[t]{2}{*}{ Asami, Y } & $605,611,719,818$ & Chong, KM & \\
\hline & & Christianson, DW & \\
\hline Babnigg, G & 731 & Chukwudi, CU & \\
\hline Bachmann, BO & 327 & Cicek, AC & \\
\hline Balunets, DV & 422 & Cichewicz, RH & \\
\hline Banerjee, $\mathrm{K}$ & 294 & Cimmino, A & \\
\hline Bang, CS & 183 & Clancy, S & \\
\hline Basher, MA & 843 & Cordero-Ampuero, J & \\
\hline Bedir, E & 51,111 & Cragg, G & \\
\hline Berestetskiy, A & 9 & Crawford, JM & \\
\hline Bergen, PJ & 415 & Creek, DJ & \\
\hline Berhow, MA & 637 & Crnovcic, I & \\
\hline Bhakta, S & 843 & Cuevas, C & \\
\hline $\mathrm{Bi}, \mathrm{B}$ & 169 & & \\
\hline Bigelow, L & 731 & Dairi, $\mathrm{T}$ & \\
\hline Biggs, TD & 313 & Daletos, G & \\
\hline Bills, GF & 141 & Dalinova, A & \\
\hline Bingman, CA & 731 & Dan, S & \\
\hline Bischoff, KM & 637 & Dawson, MJ & \\
\hline Blodgett, JAV & 15 & Deguire, SM & \\
\hline Boakes, S & 850 & De la Calle, F & \\
\hline Bolshakova, TN & 422 & Del-Prado, G & \\
\hline Boonlarppradab, C & 459,511 & Dezhenkova, LG & \\
\hline Boulos, RA & 879 & Ding, $N$ & \\
\hline Boutoille, D & 806 & Dobrynina, OY & \\
\hline Bowman, M & 524 & Doi, Y & \\
\hline Bowman, MJ & 637 & Dorrestein, PC & \\
\hline Braña, AF & 179 & Dowd, PF & \\
\hline Bretonnière, C & 806 & $\mathrm{Du}, \mathrm{D}$ & \\
\hline Brockman, AA & 327 & Duzgun, AO & \\
\hline Brogan, DM & 73 & & \\
\hline Brucoli, F & 843 & Earl, D & \\
\hline $\mathrm{Bu}, \mathrm{Y}-\mathrm{Y}$ & 57 & Eguchi, T & 287,5 \\
\hline Budak, A & 825 & Ekiz, G & 1 \\
\hline Burkart, MD & 524 & Enquisr, JA & \\
\hline Bush, K & 600 & Esteban, J & \\
\hline
\end{tabular}

Evangelopoulos, D
Evidente, A

843

811

169

731

731

467

631

415

104

141

765

3,811

149

104

273

164

327

486

622

858

871

9
71

686

580

616

415

731

388

119

702

33

850

327

388

686

549

773

422

415

353

631

811

858

327

7, 541

111

331

686

Fenical, W

Fernández, R

Fernandez-Roblas, R

Fletcher, MH

Florez, FK

Forrest, A

Fox, KR

Foxley, MA

Friedline, AW

Friestad, GK

Frisvad, JC

Fujimori, M

Fujisawa, N

Fujita, N

Fujiwara, M

Fukaya, K

Fukuda, I

Fukumoto, A

Fukushima, M

Furman, B

Furuta, T

Fushimi, H

Futamura, Y

Ge, M

Gintsburg, AL

Glatzhofer, DT

Gloer, JB

Gómez-Barrena, E 686

Good, L

Grkovic, T

Groot, H

Grzeszczyk, B

Gu, Q

Guan, X

Guitton, C

Guo, L

Guo, Q

Guzman, JD

Hamada, K

Hamada, M

Hamashima, Y

Hameş, EE

Han, L

Han, X

Hanaki, $\mathrm{H}$

Harunari, E

Hashizume, $\mathrm{H}$

Hassan, HM

Hayakawa, Y
511, 582

344, 780

\section{3}

415

843

871

871

294

141

119

133

712

747

273

776

395

541

164

299

368,440

4, 456

62

422

871

631

686
622

594

783

164

467

153

806

153

173

843

287

26, 159, 712

299

111

773

769

319, 605

159, 403

890, 893

511 


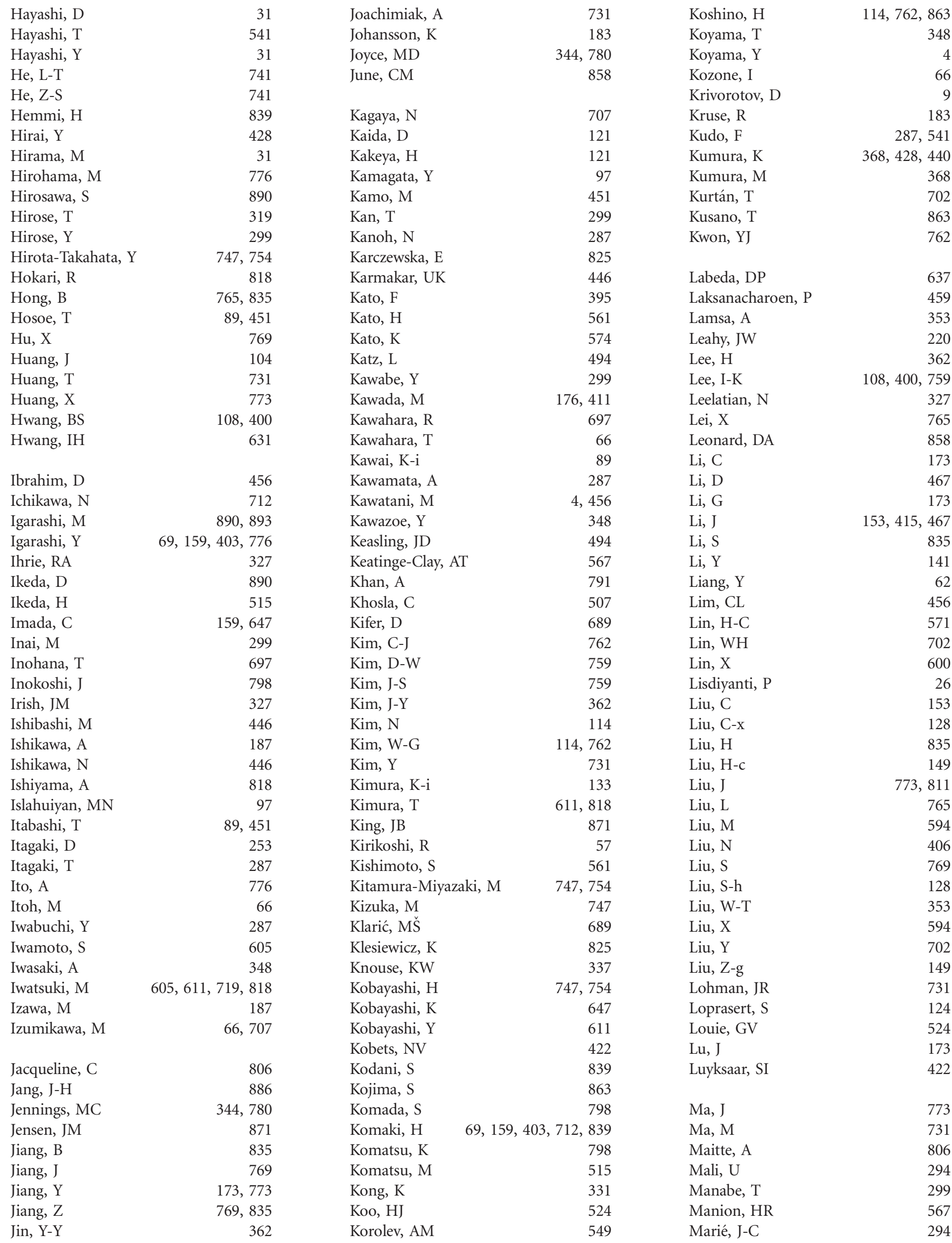




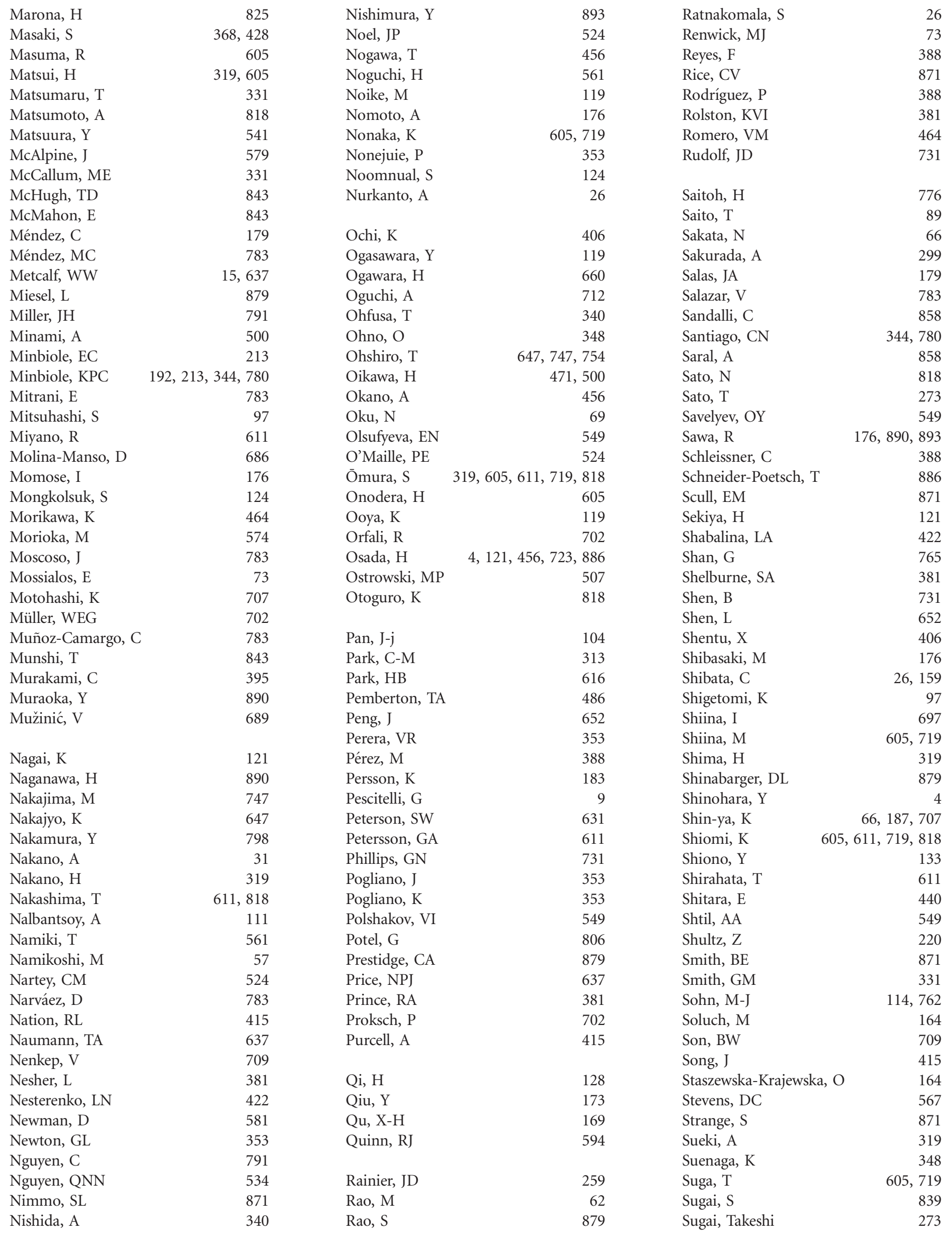




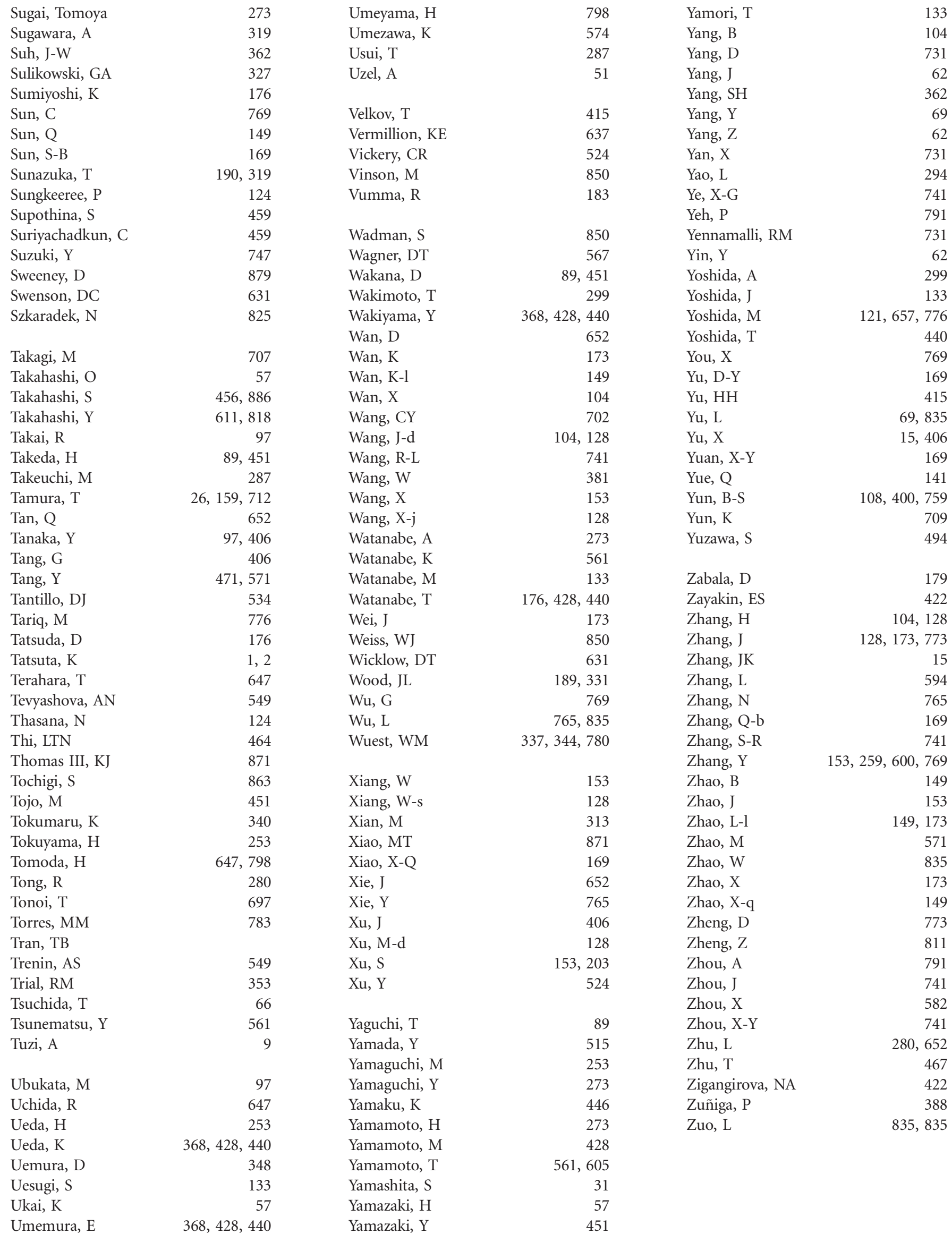

